\title{
Miranda
}

Revue pluridisciplinaire du monde anglophone /

Multidisciplinary peer-reviewed journal on the English-

speaking world

1 | 2010

Variations on Darwin

Mise en scène de la métamorphose du corps dans Clarissa; Or, the History of a Young Lady (1747-48) de Samuel Richardson

\section{Hélène Dachez}

\section{OpenEdition}

\section{Journals}

Édition électronique

URL : http://journals.openedition.org/miranda/406

DOI : $10.4000 /$ miranda.406

ISSN : 2108-6559

\section{Éditeur}

Université Toulouse - Jean Jaurès

\section{Référence électronique}

Hélène Dachez, " Mise en scène de la métamorphose du corps dans Clarissa; Or, the History of a Young Lady (1747-48) de Samuel Richardson », Miranda [En ligne], 1 | 2010, mis en ligne le 23 mars 2010, consulté le 16 février 2021. URL : http://journals.openedition.org/miranda/406 ; DOI : https://doi.org/ $10.4000 /$ miranda. 406

Ce document a été généré automatiquement le 16 février 2021.

\section{c)}

Miranda is licensed under a Creative Commons Attribution-NonCommercial-NoDerivatives 4.0

International License. 


\title{
Mise en scène de la métamorphose du corps dans Clarissa; Or, the History of a Young Lady (1747-48) de Samuel Richardson
}

\author{
Hélène Dachez
}

1 En employant l'adjectif «dramatic » dans le postscript de Clarissa, Samuel Richardson propose une définition générique complexe de l'ouvrage dont il vient de conclure l'intrigue. ${ }^{1}$ L'adjectif anglais "dramatic», faux-ami partiel, qui selon le contexte, signifie dramatique, théâtral ou spectaculaire, insiste sur les qualités visuelles d'un roman entièrement épistolaire, qui regorge d'effets théâtraux et scéniques, d'artifices et de mises en scène variés. ${ }^{2}$ L'ouvrage illustre de diverses manières et à plusieurs niveaux la définition de la "mise en scène» en tant qu' « organisation matérielle de la représentation » qui concerne la " place, les « mouvements », les « jeux des acteurs" et le "choix des décors $» .^{3}$ Ces mises en scène sont régulièrement organisées par Lovelace, protagoniste qui se compare à Ovide, maitre des métamorphoses, et que Clarissa associe à Protée, dieu marin qui avait reçu de son père Poséidon le don de changer de forme à volonté. ${ }^{4}$

2 L'écriture épistolaire et l'échange de lettres entre rédacteur et récepteur sont également liés aux notions de mise en scène et de métamorphose car, si l'on écrit à quelqu'un parce qu'il est absent, l'une des fonctions de l'écriture est précisément de transformer l'absence en présence et de représenter le corps absent comme s'il était sur le devant de la scène (romanesque). Comme l'analyse Jean Rousset, « le destinataire absent [...] est présent de tout son poids [dans l'échange épistolaire]. Avec une absence créer une présence, tel est bien le pouvoir paradoxal [...] de la lettre $\%^{5}$ En même temps que l'épistolarité métamorphose l'absent en présent, le message écrit dans l'isolement du boudoir devient dialogue. ${ }^{6}$ Grâce à la technique épistolaire inventée par Richardson (« writing to the moment») dont Belford analyse les avantages, le rédacteur a en outre 
la possibilité de rendre compte des événements et des sentiments passés comme s'ils étaient en train de se dérouler ou d'être vécus :

[writing] in the midst of present distresses! How much more lively and affecting, for that reason, may her style be, than all that can be read in the dry, narrative, unanimated style of persons relating difficulties and dangers surmounted [...] the relater unmoved by his own story, how then able to move the hearer or reader? (1178). ${ }^{7}$

3 L'émotion transmise par l'intermédiaire du message et ressentie par le lecteur (aussi bien intra- qu'extra-diégétique) est d'autant plus profonde que, grâce à cette écriture très précise, le rédacteur a la possibilité de donner de nombreux détails (entre autres sur le corps des personnages). Ces précisions étaient d'autant plus facilement identifiables par les contemporains de Richardson que ces derniers vivaient dans une société et à une époque où les gestes du corps et les expressions du visage étaient codifiés, comme l'illustrent par exemple les gravures exécutées et commentées par Charles Le Brun. ${ }^{8}$ Clarissa attire l'attention sur la mise en scène rendue possible par cette écriture qui fait la part belle au visuel lorsqu'elle adresse à Anna la remarque suivante, dans des propos à la fois métafictionnels et métathéâtraux :

you will always have me give you minute descriptions nor suffer me to pass by the air and manner in which things are spoken that are to be taken notice of; rightly observing that air and manner often express more than the accompanying words (42).

4 Les moindres changements physiques doivent être dûment consignés par le rédacteur pour que le lecteur puisse se représenter la scène aussi précisément que si elle se déroulait sous ses yeux. ${ }^{9}$ Lovelace décrit ainsi avec force détails et de manière très visuelle les manifestations physiques qu'engendre sur le corps de Clarissa (et à son corps défendant) sa première demande en mariage :

oh how the mantled cheek, the downcast eye, the silent, yet trembling lip, the heaving bosom, a sweet collection of heightened beauties, gave evidence that the tender was not mortally offensive! (425).

5 La mise en scène fait partie intégrante de l'écriture romanesque et, dans une lettre qu'il écrit à Edward Moore, Richardson demande à son lecteur de se représenter les derniers instants de Lovelace: «see the voiture, tho' moving slowly, by its motion getting his wounds bleeding afresh. [...] See him giving directions. [...] See him living over the night $»^{10}$

6 Avant cette métamorphose ultime et irrémédiable, Lovelace, acteur hors-pair, joue avec son corps et épouse les diverses identités qu'il se crée avec une telle facilité que Clarissa le juge plus habile à se métamorphoser que le caméléon..$^{11}$ Lovelace parvient à devenir autre sans aucune difficulté et avec un plaisir certain car, comme le dit l'héroïne, " he is so much of the actor that he seems to enter into any character; and his muscles and features appear under obedience of his wicked will» (1003)..$^{12}$ Il n'est pas surprenant que l'un des adjectifs les plus utilisés pour décrire le libertin soit « various » $(128,445$, 462), qui insiste sur le tourbillon identitaire qu'il crée volontairement pour parvenir à ses fins auprès de Clarissa. La force de sa mise en scène consiste soit à garder sa propre identité mais à agir de façon contradictoire (si bien que Clarissa ne parvient jamais à savoir qui il est vraiment, et si elle peut avoir réellement foi en ses promesses de réformation) soit à brouiller les signes en empruntant l'identité d'autrui.

7 Lorsqu'il montre à l'hérö̈ne son bras marqué par une cicatrice qu'il a reçue au cours d'un duel, à la suite duquel il a sérieusement envisagé d'abandonner ses pratiques 
libertines (444), Lovelace révèle qu'il considère son corps comme un instrument qu'il peut utiliser à sa guise et mettre en scène en en révélant les parties d'ordinaire cachées, pour susciter l'émotion de sa spectatrice. De même, il parvient à convaincre Mrs Moore, chez qui Clarissa s'est réfugiée pour lui échapper, de lui louer une chambre grâce à un stratagème corporel aussi éculé qu'efficace : « [I] rubbed my eyes till I supposed them red» (786). L'émotion qu'il affiche sur son corps (que le lecteur sait être fausse mais que la femme croit réelle) a pour fonction de susciter la compassion de la logeuse, d'abord méfiante, si bien que grâce à cette mise en scène élémentaire, il parvient à se rapprocher de sa future victime. La scène où il prend de l'ipécacuanha est plus complexe : elle montre que la métamorphose du corps de Lovelace entraîne celle de Clarissa, qui découvre quels sentiments elle éprouve pour le roué. Lovelace ingère le vomitif (il métamorphose un corps sain en corps malade) pour voir comment l'héroïne, qui ne manquera pas de le croire souffrant, réagira. "If she has humanity, she will be concerned. But if she has love [...] it will [...] come forward, and show itself; not only in her eye, but in every line of her sweet face » (673), prédit-il à Belford..$^{13}$

8 La mise en scène de la métamorphose du corps apparaît avec le plus d'éclat lorsque Lovelace rend compte à Belford des divers déguisements qu'il revêt. Les rôles que joue le libertin sont multiples, ainsi que l'illustre la longue liste des déguisements qu'il emprunte, dont il rend toujours un compte très précis à son lecteur, à qui il dévoile les secrets de ses transformations physiques. ${ }^{14}$ Tel un acteur avant de monter sur scène, il décrit les accessoires dont il se pare (une redingote qu'il emprunte à son cocher, une perruque poudrée, une cape et des bas grossiers [766]) pour se transformer en vieil homme issu du peuple et atteint de goutte, dont il parfait la représentation en s'appuyant sur une canne et en imitant la claudication. L'aristocrate n'hésite pas à déroger à sa classe pour se modeler un nouveau corps, antithétique au sien, sur lequel convergent tous les regards. ${ }^{15}$ Dans le fiacre qui le conduit chez les Smith, humbles commerçants de gants londoniens chez qui Clarissa s'est réfugiée, le libertin répète le rôle qu'il entend jouer tout en parlant à son corps :

I charged my eyes to languish and sparkle by turns: I talked to my knees, telling them how they must bend; and in the language of a charming describer acted my part in fancy, as well as spoke it to myself (1209).

9 Il commence par se faire passer pour un juge de paix venu fouiller la demeure des commerçants. Ensuite, une fois reconnu, il joue le rôle d'un client, puis celui du vendeur dans le but de gagner la confiance des Smith qui, s'imagine-t-il, le laisseront alors aller et venir chez eux comme bon lui semble (1214-5).

10 Si Lovelace cherche à être maître de son corps, à le mettre en scène pour tromper autrui et séduire Clarissa, et s'il annonce qu'il ne retirera son masque que lorsqu'il sera sûr de parvenir à ses fins grâce à ses talents d'acteur (610), il tente aussi de commander le corps des autres (en le métamorphosant), si bien que d'acteur, il devient metteur en scène, et se montre même si habile dans l'organisation des mises en scène que les autres personnages agissent pour lui sans s'en rendre compte :

[the Harlowes] are all working for me, like so many underground moles; and still more blind than the moles are said to be, unknowing that they did so. I myself, the director of their principal motions (387).

11 A de nombreuses reprises, ces trois fonctions (d'acteur et de metteur en scène reconnu ou non) coïncident et l'incarnation simultanée de plusieurs instances dramatiques illustre le fantasme de toute puissance du roué. Le corps d'autrui s'apparente ainsi à une enveloppe vide, malléable à souhait, que Lovelace modèle à sa guise. Tomlinson, 
personnage créé de toutes pièces par le libertin pour faire croire à Clarissa que sa famille veut se réconcilier avec elle et l'a choisi comme intermédiaire, se compare à de la pâte («dough ») capable de prendre la forme que veut lui donner Lovelace. ${ }^{16}$ Deux autres métaphores récurrentes illustrent combien Lovelace parvient à manipuler le corps d'autrui. En écho à l'ouvrage de La Mettrie, l'organisme devient une machine dont la seule fonction est d'obéir aux directives du libertin $(275,747) .{ }^{17}$ La seconde métaphore est celle de la marionnette, dont Lovelace tient les ficelles et dirige tous les mouvements, comme il l'explique au sujet du frère de Clarissa : « cooling, or inflaming his [...] violent passions, as may best suit my purposes [...] shall enable me to dance his employer upon my own wires" (144-5). ${ }^{18}$ A l'inverse, il est révélateur que Lovelace refuse d'être le pantin qu'il craint de devenir s'il se réforme et épouse Clarissa :

what the devil had she to do to let her fancy run agadding after a rake ? [...] / oh but truly, she hoped to have the merit of reclaiming him. She had formed pretty notions how charmingly it would look to have a penitent of her own making dangling at her side to church, through an applauding neighbourhood (970).

Le maître des métamorphoses ne peut envisager que son corps soit mis en scène par autrui.

13 Avant de présenter sur scène les corps qu'il a métamorphosés, Lovelace se livre à des séances de répétition théâtrale au cours desquelles il règle les mouvements des acteurs et corrige leur posture, comme le fait un metteur en scène. Les impératifs qu'il adresse à Joseph Leman, valet des Harlowe en réalité à sa propre solde, révèlent que le roué décide des moindres gestes de son homme de main, dont il transforme la démarche : " observe to keep at a distance that [Clarissa] may not discover who you are. Take long strides, to alter your gait; and hold up your head » (384). De même, le libertin, avant de mettre sur la même scène Clarissa et deux prostituées censées être sa tante et sa cousine, leur fait répéter les rôles qu'il a créés pour elles. Hors contexte, les propos de Lovelace pourraient être ceux qu'adresse un metteur en scène à ses acteurs :

let not your faces arraign your hearts [...]

A little graver, Lady Betty. More significance, less bridling, in your dignity.

That's the air! Charmingly hit-Again-You have it. [...]

Now for your part, cousin Charlotte! - [...]

Those glances will never do. A down-cast bashful turn, if you can command it-Look upon me. Suppose me now to be my beloved. [...]

Sprightly, but not confident, cousin Charlotte!-Be sure to forget not to look down, or aside, when looked at. When eyes meet eyes, be yours the retreating ones (876).

Une fois devant Clarissa, les prostituées-actrices jouent leur rôle avec tant de conviction qu'elles parviennent à persuader l'héroïne de retourner chez la Sinclair pour y chercher ses vêtements, et l'on sait que le viol a lieu chez la tenancière peu après. ${ }^{19}{ }^{\prime}$ 'est en outre un véritable code gestuel que Lovelace élabore pour indiquer à Tomlinson comment se comporter face à l'héroïne et comment conduire la conversation. Les signes doivent être assez clairs pour que l'homme de main puisse les comprendre et en tenir compte, mais assez discrets pour que Clarissa ne les voie pas. Le libertin explique à Belford le sens de ce corps devenu un ensemble de signes que Tomlinson doit décoder :

a wink of the left eye was to signify, Push that point, captain. / A wink of the right, and a nod, was to indicate approbation of what he had said. / My forefinger held up, and biting my lip, Let off that as fast as possible (832). 

d'autrui révèle sa volonté de posséder totalement les corps, comme l'illustre la scène de l'enlèvement, où le libertin enlace Clarissa pour l'amener à lui faire quitter le domaine paternel avec un empressement grandissant et une violence à peine contenue. ${ }^{20} \mathrm{~A}$ son degré ultime, la possession devient ingestion, et il n'est pas fortuit que les personnages utilisent l'image du parasite et celle de l'araignée tissant sa toile autour de sa victime pour décrire leur relation. ${ }^{21}$ Dans le septième papier qu'elle rédige peu après le viol, Clarissa compare son bourreau à deux parasites, qui se nourrissent de sa substance et vident son enveloppe charnelle au point de causer sa mort: "thou pernicious caterpillar that preyest upon the fair leaf of virgin fame [...] thou eating cankerworm that preyest upon the opening bud » (892). La mise en scène de la métamorphose du corps apparaît dans toute sa force lorsque Lovelace se représente en araignée jouissant du spectacle de sa victime, transformée en insecte immobilisé dans la toile qu'il a tissée : « out steps the [...] tyrant, winds round and round the poor insect [...] and when so fully secured, that it can neither move leg nor wing, suspends it, as if for a spectacle to be exulted over $"(418)$.

Si le corps de Clarissa, ici, menace de disparaître, Lovelace s'imagine à plusieurs reprises et dans diverses mises en scène qu'il se multiplie. Ce fantasme de paternité est par exemple à l'origine de la vision où il se représente Clarissa donner le sein à deux enfants mâles :

[I see] a twin Lovelace at each charming breast [...]. / I now, methinks, behold this most charming of women in this sweet office, pressing with her fine fingers the generous flood into the purple mouths of each eager hunter by turns (706). ${ }^{22}$

Plus tard, il voit en rêve les corps se métamorphoser selon le principe de l'antithèse, et se multiplier : la douairière qui recueille Clarissa enfuie se transforme en " Mother $\mathrm{H}$ ", vieille femme qui aide Lovelace, puis en jeune femme, et les scènes se succèdent à un rythme effréné jusqu'à ce que le songe s'achève sur plusieurs naissances (918-922).

Le fantasme de paternité et la multiplication des corps révèlent un renversement du rapport de force : le libertin, qui s'imaginait Clarissa cernée par des Lovelace dans tous les coins («Lovelaces in every corner » [765]), devient obsédé par sa victime, si bien qu'il croit la voir partout alors qu'elle vient de s'enfuir de chez la Sinclair. L'esprit troublé de Lovelace métamorphose l'absence en présence spectrale :

from her room to my own [...] and in and out of every place where I have seen the beloved of my heart, do I hurry; in none can I tarry; her lovely image in every one [...] rushing cruelly upon me (740).

En réponse inversée à la pénétration physique effectuée lors du viol, c'est Clarissa qui semble avoir envahi le corps de Lovelace et qui s'exprime à travers lui grâce à un phénomène proche de la ventriloquie, dont il rend compte à Belford en joignant le geste à la parole : «she has a friend here (clapping my hand on my breast) that pleads for her with greater and more irresistible eloquence, than all the men in the world can plead for her » (837). ${ }^{23}$ Se sentant vidé de sa substance une fois que Clarissa s'est enfuie, il devient lui-même fantôme et envisage de hanter les églises et la demeure des Smith, où il croit que l'héroïne peut se trouver : 
I would go round to all the churches in London and Westminster, when there were prayers or service, from sunrise to sunset and haunt [the Smiths'] house like a ghost till I had the opportunity my soul panted after (1222). battu à son propre jeu, si bien que ses mises en scène deviennent des mascarades qu'il ne parvient pas à maîtriser. Bien qu'il adresse à Tomlinson le signal lui indiquant dans quel sens poursuivre la conversation, l'homme de main éprouve du remords à tromper Clarissa et refuse d'obéir au corps-signe de son maitre. ${ }^{24}$ Le scénario élaboré par Lovelace, qui envisage de faire de son histoire avec Clarissa une comédie qu'il intitulera The Quarrelsome Lovers (571), devient impossible à mettre en scène, et la comédie prévue devient tragédie réelle. Les scénarios envisagés se métamorphosent en leur contraire, et tout semble échapper au libertin, comme l'analyse Serge Soupel au sujet du viol, acte qui « est l'effet d'une brutalité imposée par les circonstances, encouragée par la Sinclair et par son entourage, [et] que ne prépare aucunement la finesse extrême des calculs patients du chasseur $» .25$ de manière révélatrice, à la métamorphose du corps. Grâce au bestiaire, Lovelace, qui se voyait aigle (559) et éléphant capable de toucher les cieux avec sa trompe (473), devient un vieux hérisson pourchassé, dont Mowbray décrit l'attitude de démission: "[he] creeps into holes and corners like an old hedgehog hunted for its grease » (1360). Tout comme ses acolytes finissent par refuser de lui obéir ou lui imposent leur volonté, Lovelace perd la maîtrise de son propre corps, qui agit autrement qu'il ne l'avait escompté, si bien que le roué se demande, juste avant de conduire sa future victime chez la Sinclair :

What makes my heart beat so strong? Why rises it to my throat in such halfchoking flutters? [...] 'Tis a plotting villain of a heart: it ever was; and ever will be, I doubt [...] I [am] so little its master! (520).

Le cœur de Lovelace semble imposer à ce dernier sa volonté propre, contrecarrer la mise en scène préparée par le libertin, et épouser la cause de Clarissa. ${ }^{26}$ Dans des termes qui font la part belle à la métathéâtralité, Lovelace est conscient qu'il a élaboré un scénario qu'il est incapable de jouer: "I have overplotted myself. [...] My divine Clarissa has beat me out of my play » $(574,717)$.

L'adjectif "divine » que Lovelace applique à sa victime est profondément révélateur, car il consacre la présence de l'ironie dramatique que Richardson exerce à l'encontre du protagoniste et annonce le parcours de l'héroïne. Dès le début du roman, Lovelace, guidé par l'hubris, tel Pygmalion, exprime sa volonté d'aller plus loin que ne l'ont fait les auteurs qu'il a lus et de métamorphoser Clarissa en déesse: " these confounded poets [...] fired my imagination and set me upon a desire to become a goddess-maker. [...] I must create beauty and place it where nobody else will find it " (143). Clarissa devient, certes, déesse à cause de (ou plutôt grâce à) lui, mais l'ironie dramatique veut que ce soit justement cette métamorphose qui sépare les deux personnages à jamais et qui permette à l'héroïne de nier son corps, sur lequel le libertin n'a finalement aucune prise. ${ }^{27} \mathrm{Si}$ Lovelace entend effectuer la mise en scène de la métamorphose des corps par le jeu et par la théâtralisation, Clarissa le fait par une ascèse qui la conduit à transcender le corps. Comme l'analyse Maurice R. Funke, « [they are] mirror reflections to each other. [...] She chooses nihilism, and he chooses megalomania $»{ }^{28}$ 
rêve (Lettre 417), Clarissa est en ascension constante, comme dans la scène où elle rejette violemment le libertin et lui lance : «my soul is above thee, man! [...] Urge me not to tell thee how sincerely I think my soul above thee! » (646). ${ }^{30}$ La transformation du corps de Clarissa prend ainsi la forme d'une catharsis charnelle, processus métamorphique qui illustre les propos de Jean-Pierre Albert, selon qui « une femme ne devient sainte qu'au terme d'une purification hyperbolique [d'une] sorte d'élision du sang $"{ }^{31} \mathrm{Il}$ est à remarquer qu'une fois le processus de transcendance commencé, la jeune fille ne porte plus que des vêtements blancs $(889,1064,1345)$, en écho à l'Apocalypse de Jean (7:13-17). ${ }^{32}$ Clarissa se construit un personnage de sainte et organise sa mort avec une sérénité remarquable, que Richardson fait contraster avec les angoisses dont Belton et la Sinclair font l'expérience lors de leur trépas. Belford, qui est le seul personnage à assister aux trois scènes de mort, est fasciné par la quiétude de l'héroïne : «the calmness and serenity with which she can talk of death, and prepare for it, as if it were an occurrence as familiar to her as dressing and undressing » (1276). $\mathrm{Au}$ contraire, les deux épisodes qui encadrent le décès de Clarissa montrent des corps meurtris par les soins endurés, morcelés par les amputations et déformés par la douleur $(1230,1241)$. La chute dont est victime la Sinclair (qu'il convient de lire comme la rétribution de ses actes infâmes) a pour conséquence de transformer la tenancière en créature monstrueuse, dotée des caractéristiques du loup et de la salamandre: « howling more like a wolf than a human creature » (1387), « her big eyes goggling and flaming-red as we may suppose those of a salamander " (1388). L'organisme de la Sinclair, que décrit Belford en termes qui insistent sur les transformations corporelles, devient, à l'approche de la mort, difforme et difficilement reconnaissable : « her wide mouth [...] splitting her face, as it were, in two parts » (1388)..$^{33}$

Alors que les autres corps se corrompent et se décomposent, celui de Clarissa reste identique à lui-même, ou, plus exactement, si l'on pense à l'étymologie du terme, intact. Cet état est éminemment paradoxal pour un organisme qui a été soumis à un viol, acte censé transformer de manière a priori irrémédiable le corps de la vierge Clarissa. L'outrage, d'ailleurs, ne signifie pas la réussite du libertin, puisque, à cause de 
l'opiat que lui a fait boire la Sinclair, Clarissa ne s'est rendu compte de rien. Comme l'analyse James Carson, « [the rape is] an inversion of the rake's fantasy of animation. The Pygmalion fantasy is answered and frustrated by an elided representation of quasinecrophilia $»{ }^{34}$ Lovelace regrette que Clarissa, en ne réagissant pas lors de l'outrage, se soit jouée de la métamorphose charnelle qu'il comptait effectuer :

I had rather [...] she should have retained all her active powers, though I had suffered by her nails and teeth, than that she should be sunk into such a state of absolute-insensibility (886).

C'est grâce à une néantisation de l'événement ou, pour citer Freud, "[un] effort d'annulation rétroactive [par lequel l'événement est traité] comme "non arrivé" ", que l'héroïne peut métamorphoser son corps à rebours et trouver par là même un moyen de nier les conséquences physiques du viol. ${ }^{35}$ Le mouvement ascensionnel vers Dieu se combine alors à l'immobilité du corps de Clarissa, comparé à la glace (413), à une statue (830), et à une pierre qui se durcit au contact de l'air si bien qu'il est impossible de lui faire subir la moindre transformation..$^{36}$

Un jeu sur la monstration et sur l'ellipse vient compléter l'échec de la métamorphose charnelle que Lovelace tente d'effectuer sur le corps de Clarissa et le retournement paradoxal que cette dernière parvient à accomplir. Le souhait le plus cher de l'héroïne, après le viol, est de disparaître, comme elle le précise à son bourreau : «let me be carried out of [...] your sight, and let me be put into that Bedlam privately [...] don't let me be made a show of " (895). Or, le corps-oxymore de Clarissa, devenu un ravissant squelette et un ravissant cadavre (" [a] lovely skeleton » [1231], «[a] lovely corpse » [1367]) exerce une fascination indéniable. L'héroöne exploite cet attrait dans son testament, en permettant aux membres de sa famille de voir sa dépouille, mais en interdisant à Lovelace de poser les yeux sur elle, sauf s'il le désire vraiment (1413). Grâce à ses dernières volontés, elle garde la mainmise sur un corps qu'elle a su métamorphoser pour effacer l'outrage que lui a fait subir Lovelace et dont elle autorise ou non, selon les spectateurs, le spectacle, corps d'autant plus attirant et fascinant qu'elle meurt en odeur de sainteté. Comme l'explique Belford

We could not help taking a view of the lovely corpse, and admiring the charming serenity of her noble aspect. The women declared they never saw death so lovely before; and that she looked as if in an easy slumber, the colour having not quite left her cheeks and lips (1367).

31 La métamorphose scelle non seulement le destin de l'héroïne, mais aussi celui du roman. Au début de son parcours, Clarissa, qui se sait incapable de se faire entendre des membres de sa famille, se compare à une plume soumise au moindre souffle de vent. ${ }^{37}$ Or, ce corps-plume se métamorphose en corps-texte, régi par la plume de la rédactrice, qui répartit les lettres (1198), organise l'ouvrage qui raconte son histoire, et demande à Belford de composer deux exemplaires qu'il fera circuler (1418), si bien que de façon concomitante à l'ensevelissement du corps physique de Clarissa apparaît le corps textuel de Clarissa. Robert A. Erikson analyse ce processus compensatoire par lequel la chair se métamorphose en un texte destiné à être lu/vu par le plus grand nombre : "she translates herself into her own Scripture. [...] She is the flesh made word.$^{38}$ L'organisme de Clarissa, qui refusait de se mouvoir, de parler à Lovelace et d'apparaître en public lors d'un procès contre lui (1254-55), se transforme en corps textuel, éloquent, mobile, et qui racontera à tous les lecteurs l'histoire de Clarissa.

Les métamorphoses du corps, dont l'auteur propose diverses mises en scène, sont ainsi au cœur du roman de Richardson. Replacer l'ouvrage dans le contexte socio-politique 
de son époque permet d'interpréter le triomphe de Clarissa (qui compense les souffrances physiques qu'elle a endurées par l'élaboration d'un texte), et l'échec de Lovelace (qui, malgré la théâtralisation de son propre corps et de celui d'autrui, ne parvient pas à inscrire de manière durable dans l'organisme de sa victime la marque de la métamorphose qu'il cherche à opérer) comme le signe de la décadence de l'aristocratie et de l'essor de la bourgeoisie. ${ }^{39}$ Clarissa, roman protéiforme où théâtre et mise en scène jouent un rôle capital, rend compte des profonds changements qui s'effectuent dans l'Angleterre du XVIIIe siècle et montre que la métamorphose concerne tout ensemble le corps physique, le corps textuel et le corps social.

\section{BIBLIOGRAPHIE}

Albert, Jean-Pierre. Le Sang et le ciel : les saintes mystiques dans le monde chrétien. Paris : Aubier, 1997.

Bynum, Caroline Walker. Holy Feast and Holy Fast: The Religious Significance of Food to Medieval Women. Berkeley: University of California Press, 1987.

Carroll, John (ed.). Selected Letters of Samuel Richardson. Oxford: Clarendon, 1964.

Carson, James. « Narrative Cross-Dressing and the Critique of Authorship in the Novels of Richardson. » In Writing the Female Voice: Essays in Epistolary Literature. Ed. Elizabeth C. Goldsmith. Boston: Northeastern University Press, 1989. 95-113.

Doody, Margaret Anne. « Disguise and Personality in Richardson's Clarissa. » Eighteenth-Century Life 12 (1988): 18-39.

Erikson, Robert A. " 'Written in the Heart': Clarissa and Scripture. » Eighteenth-Century Fiction 2 (1989): 17-52.

Freud, Sigmund. Inhibition, symptôme et angoisse. Michel Tort trans. 1926. Paris : PUF, 1965.

Funke, Maurice R. From Saint to Psychotic: The Crisis of Human Identity in the Late Eighteenth Century. Frankfurt: Lang, 1983.

Glaser, Brigitte. The Body in Richardson's Clarissa. Heidelberg: Universitätsverlag, 1994.

Kinkead-Weekes, Mark. Samuel Richardson: Dramatic Novelist. London: Methuen, 1973.

\section{NOTES}

1. Samuel Richardson, Clarissa; Or, the History of a Young Lady, ed. Angus Ross (1747-48; Harmondsworth: Penguin, 1985).

2. L'on pense notamment aux conversations où les propos des personnages sont présentés au style direct et précédés de didascalies $(624,682-85)$, comme au théâtre.

3. Grand Robert de la langue française, entrées « mise ", « mise en scène ».

4. "Ovid was not a greater master of metamorphoses than thy friend", se vante Lovelace à Belford (412), et Clarissa compare son futur bourreau, dont elle ne comprend pas les agissements, 
à Protée : «I am strangely at a loss what to make of this man. He is a perfect Proteus. I can but write according to the shape he assumes at the time»; ce passage figure dans l'édition Dent (Samuel Richardson, The History of Clarissa Harlowe, ed. Ernest Rhys [1747-48; London: Dent, 1932] 4 vols, 2.82), mais pas dans l'édition Penguin. Les Métamorphoses d'Ovide sont constituées de 246 fables qui montrent des humains transformés en pierre, en végétal ou en animal, depuis la création du monde jusqu'à la métamorphose de César en étoile.

5. Jean Rousset, Forme et signification : essais sur les structures littéraires de Corneille à Claudel (1962 ; Paris : Corti, 1976) 72, 78.

6. Lovelace constate qu'écriture et conversation se correspondent, car «familiar writing is but talking» (915) ; et Clarissa, éloignée de sa confidente, se réjouit que l'écriture épistolaire lui donne la possibilité de communiquer avec elle : « conversing with you-by letter-as I cannot in person $»(53)$.

7. "[Letters] are to the moment dramatic narratives, attempts to catch living voices in a dramatic present» (Mark Kinkead-Weekes, Samuel Richardson: Dramatic Novelist [London: Methuen, 1973] 396.)

8. Charles Le Brun, Expression des passions de l'âme (1727 ; Paris : Bibliothèque Interuniversitaire de Lille, 1990). Sur la stylisation des gestes théâtraux, voir l'article de Leo Hughes, "Theatrical Conventions in Richardson: Some Observations on a Novelist's Technique ", Restoration and Eighteenth-Century Literature, Essays in Honor of Alan Dugald McKillop (William Marsh Rice University: University of Chicago Press, 1963) 239-250.

9. En lisant le message où Morden rend compte de l'enterrement de l'hérö̈ne, Belford écrit : " your last letter so naturally describes all that passed that I have every scene before my eyes " (1409).

10. John Carroll, ed. Selected Letters of Samuel Richardson (Oxford: Clarendon, 1964) 122.

11. «Don't think me the changeable person, I beseech you », prie-t-elle Anna, « [...] he is a perfect chameleon; or rather more variable than the chameleon; for that, it is said, cannot assume the red or the white; but this man can. And though black seems to be his natural colour, yet has he taken great pains to make me think him nothing but white " (édition Dent, 2.82).

12. "This man has very ready knees" (166), constate Clarissa, qui l'appelle aussi «the ready kneeler » (379). Lovelace lui-même se vante à Belford du fait que « every nerve and fibre of me is always ready to contribute its aid, whether by health or by ailment, to carry a resolved-on roguery into execution $»(676)$.

13. Lovelace interprète le trouble que ressent Clarissa à la vue de ce corps malade avec une facilité déconcertante: "I see I have gained my end. I see the dear soul loves me. I see she forgives me all that's past. I see I have credit for a new score » (677).

14. Selon Mark Kinkead-Weekes, « his inveterate habit of dramatizing himself, and everything around him [shows the] ever shifting kaleidoscope of a mind endlessly role-playing, and shifting roles in mid-stream or mid-sentence » (438-39).

15. Margaret Anne Doody écrit à ce sujet : « like a Launcelot of old [...] he is willing for his lady's sake to undergo the ordeal not only of discomfort but of dishonour, of class alteration " (« Disguise and Personality in Richardson's Clarissa », Eighteenth-Century Life 12 [1988]: 18-39) 18. Plus tard, il envisage de se déguiser en prêtre pour pouvoir s'approcher de sa victime (1144).

16. «I am dough in your hands, to be moulded into what shape you please » (838).

17. Julien Offray de La Mettrie, L'Homme-machine, L'Homme-machine suivi de L'Art de jouir (1748; Paris : Bossard, 1921) 1-147.

18. Le libertin reprend l'image pour écrire, au sujet de la confidente de Clarissa : «Miss Howe herself is but a puppet danced upon my wires » (464).

19. Clarissa comprend bien plus tard seulement que les deux femmes ont interprété un scénario élaboré par Lovelace : « she had her principal instructions from him, and those written down too 
[...] for I have recollected since, that I once saw this Lady Betty [...] take out a paper from her stays and look into it, and put it there again » (999).

20. «He kept drawing me after him » (374), dit Clarissa, qui décrit les gestes du libertin en détail, "putting his arm around me and again drawing me with gentle force after him » (375), «then another push, and another [...] I ran as fast as he [...] my fears [...] adding wings to my feet » (380). 21. L'édition Dent contient une lettre absente de l'édition Penguin, où Lovelace envisage d'enlever Anna Howe et sa mère, ainsi que leur bonne, pour les violer, lors de leur voyage dans l'Ile de Wight. Il s'agit de la lettre 208 (2.418-425), où Lovelace parle de «three such enormous rapes» (2.421).

22. Voir aussi la remarque suivante : «she ought to have forgot [the rape] by this time, except the charming, charming consequence happen, that still I am in hopes will happen » (916), et le fait qu'il imagine que Clarissa mettra au monde tous les ans un enfant mâle (720).

23. Un peu plus loin, Lovelace se plaint que Clarissa lui vole sa plume, de sorte qu'il ne peut plus écrire ce qu'il veut : « she had stolen my pen [...] and thus she wrote with it, in a hand exactly like my own » (848).

24. "He should have been quite angry-for what gave I him the nod positive? He should have stalked to the window, as for his whip and hat» (833), s'indigne Lovelace en voyant que Tomlinson ne suit pas son scénario.

25. Serge Soupel, «Science et violence chez Lovelace », Savoir et violence en Angleterre du XVIe au XIXe siècle, éd. Alain Morvan (Lille : Presses Universitaires de Lille, 1987) 121-31, 128.

26. De même, Lovelace, venu chez Mrs Moore dans l'espoir de parler à Clarissa, est trahi par son cœur, qui l'oblige à se démasquer plus tôt qu'il ne l'avait prévu $(769,772)$. C'est en outre à cause d'un transport non maîtrisé qu'il demande à Clarissa de l'épouser : " an involuntary impulse, in defiance of premeditation and of all [my] proud schemes » (493).

27. "Clarissa has the greatest of Triumphs even in this World", dit Richardson dans sa correspondance personnelle, " the greatest I will venture to say, even in, and after the Outrage, and because of the Outrage that ever Woman had " (Carroll 108).

28. Maurice R. Funke, From Saint to Psychotic: The Crisis of Human Identity in the Late Eighteenth Century (Frankfurt: Lang, 1983) 79.

29. Caroline Walker Bynum, Holy Feast and Holy Fast: The Religious Significance of Food to Medieval Women (Berkeley: University of California Press, 1987) 207-209.

30. Reprenant le contraste entre le haut et le bas, le libertin écrit qu'à la suite de l'outrage, «she had been lifted up above the standards of a common mortal » (806).

31. Jean-Pierre Albert, Le Sang et le ciel: les saintes mystiques dans le monde chrétien (Paris : Aubier, 1997) 259. "Here is your glory:», lui dit Mrs Norton, " you are brightened and purified by your sufferings! » (1328).

32. Belford la décrit à Lovelace le 6 septembre, veille de sa mort : « I found her up and dressed; in a white satin nightgown » (1345).

33. "She is made into an animated gargoyle, a horrifying personification of shapeless, uncontrolled violence. (The "tradition" for this goes back to the Middle Ages, through the monsters of Spencer and Bunyan, and also directly, through the pre-latitudinarian hell-fire sermon) », analyse Mark Kinkead-Weekes (441).

34. James Carson, «Narrative Cross-Dressing and the Critique of Authorship in the Novels of Richardson ", Writing the Female Voice: Essays in Epistolary Literature, ed. Elizabeth C. Goldsmith (Boston: Northeastern University Press, 1989) 95-113, 105.

35. Sigmund Freud, Inhibition, symptôme et angoisse, Michel Tort trans. (1926; Paris : PUF, 1965) 42. 36. Selon Lovelace, l'inflexibilité de Clarissa l'apparente à la pierre : «there is a stone [...] so soft in the quarry that it may, in a manner, be cut with a knife; but if the opportunity be not taken, and it is exposed to the air for any time, it will become as hard as marble, and then with difficulty it yields to the chisel. So this lady » (844). 
37. «How am I driven to and fro, like a feather in the wind, at a pleasure of the rash, the selfish, the headstrong » (328), déplore Clarissa.

38. Robert A. Erikson, "Written in the Heart": Clarissa and Scripture ", Eighteenth-Century Fiction 2 (1989): 17-52, 46. Brigitte Glaser, qui reprend cette analyse, écrit : « [Clarissa] can replace the violated body with discourse and thus regain her old sense of integrity » (The Body in Richardson's Clarissa [Heidelberg: Universitätsverlag, 1994]) 26.

39. Pour Brigitte Glaser : «Richardson wishes to degrade the aristocratic body [...] and wishes to elevate, at the same time, the healthy, bourgeois body which will then strengthen the middle classes' claim to increased importance and influence » (75).

\section{RÉSUMÉS}

En comparant Lovelace, son personnage d'aristocrate libertin, à Ovide et à Protée, Samuel Richardson place son roman épistolaire sous le signe de la métamorphose des corps. À la fois metteur en scène de son propre corps et du corps des autres, le roué montre comment et combien le corps, objet de manipulations, de représentations, de désirs et de fantasmes, devient un enjeu dans la relation à autrui. C'est notamment le cas dans celle qu'il entretient avec Clarissa, sa future victime issue de la bourgeoisie, dont le destin tragique est porté et scellé par le corps. À la suite d'un viol quasi nécrophile, l'organisme de la jeune fille, qui s'emploie à faire taire ses besoins et ses désirs physiques, s'immobilise, se pétrifie et, au terme d'un long parcours ascétique dont la forme épistolaire du roman rend compte pas à pas, se transforme en texte : Clarissa.

Le discours que tient Richardson sur la métamorphose du corps mérite en outre d'être replacé dans le contexte socio-politique d'une époque où s'affrontent une bourgeoisie ascendante et une aristocratie déclinante, et où s'élaborent de manière concomitante de nouveaux rapports au corps.

The theatrical metamorphosis of the body is at the heart of Samuel Richardson's epistolary novel Clarissa. As the stage-manager of his own and other characters' bodies, Lovelace, the aristocratic rake, explores and discloses the processes through which the body can be staged and manipulated, at the same time as it becomes an object of desire and phantasm, and is at the core of the relationship with the other-as is best illustrated by Lovelace's relationship with Clarissa, his middle-class victim whose fate is sealed through her body. At the end of her long ascetic progress whereby flesh metamorphoses into word, Clarissa's organism, whose physical needs and desires she manages to silence, turns into a text: Clarissa.

Richardson's discourse on the staged metamorphosis of the body should also be analyzed in relation to the socio-political context of his time, when the ideology of the rising bourgeoisie was pitted against that of the declining aristocracy, and when new relations to the body emerged.

\section{INDEX}

Keywords : theatricality, metamorphosis, body, epistolarity

Mots-clés : mise en scène, métamorphose, corps, épistolarité 


\section{AUTEURS}

\section{HÉLÈNE DACHEZ}

Professeur

Université de Toulouse II-Le Mirail

helene.dachez@univ-tlse2.fr 